\title{
Fast Handoff Mechanism in Wireless Local Area Networks (WLAN) using Neighbor Graph Algorithm
}

\author{
Debabrata \\ Sarddar \\ Jadavpur University, \\ Kolkata-700032
}

\author{
Papri Mani \\ West Bengal \\ University of \\ Technology, \\ Kolkata- 700064
}

\author{
Utpal Biswas \\ University of \\ Kalyani, Kalyani, \\ Nadia, WB- 741235.
}

\author{
M.K.Naskar \\ Jadavpur University, \\ Kolkata- 700032.
}

\begin{abstract}
Rapid progress in the research and development in wireless technology has created different types of high performance and highly optimized wireless networks. They provide various types services like VoIP, multimedia, video conference, chatting etc. Unreliable and inefficient handoff procedures reduce the quality and reliability of the networks. So to maintain a certain level of Quality of Service (QoS) a seamless handoff should be ensured. Throughout the last few years there has been plenty of research aimed towards reducing handoff delay incurred in the different level of wireless communications. Here we propose a fast handoff method in which we use pre-authentication based on Neighbor Graph Algorithm.
\end{abstract}

Keywords: Handoff Delay, NG (Neighbor Graph), RSS (Received Signal Strength), Wireless Station or user end point (STA), Access point (AP).

\section{INTRODUCTION}

IEEE 802.11 based wireless local area networks (WLAN) have been widely deployed for business, research and personal applications nowadays. The most challenging issue in mobile network technology is handoff management. Quality of service (QoS) demanding applications like VoIP, multimedia, video conference, banking transactions etc need seamless handoff. Reliable and efficient handoff procedures offer a robust communication between two end points.

\section{IEEE 802.11}

IEEE 802.11 specifies [10] a set of Wireless LAN standards developed by working group 11 of IEEE LAN/MAN Standard Committee (IEEE 802). The 802.11 family includes (a-j) and $n$ standards. $802.11 \mathrm{~b}$ was the first widely accepted wireless networking standard followed by 802.11a and 802.11g. IEEE $802.11 \mathrm{~b}$ and $802.11 \mathrm{~g}$ use the $2.4 \mathrm{GHz}$ and $802.11 \mathrm{a}$ uses the 5 $\mathrm{GHz}$ band.

IEEE 802.11a, 802.11b and 802.11g specify three different PHY layers however MAC is the same in all the three. 802.11 specify:

- $\quad$ PHY sub-layer

-802.11a, 802.11b, 802.11g

- $\quad$ MAC sub-layer

- Independent of the PHY

- DCF (Distributed Coordination Function)

- $\quad$ CSMA/CA

- PCF (Point Coordination Function)

- $\quad$ MAC management.
IEEE 802.11 MAC specification allows two modes of operations: Ad- hoc and Infrastructure.

i. In Ad- hoc mode STA recognize each other by establishing a peer- 2- peer communication between them.

ii. In Infrastructure mode an Access point (AP) provides network connectivity to its associated STA and forms (Basic Service Set) BSS. Multiple APs and their associated STAs form an Extended Service Set (ESS). Besides there exits a unique level called Service Set Identifier (SSID) which characterizes all APs and STAs in WLAN. Fig.1 shows the IEEE 802.11x network infrastructure.

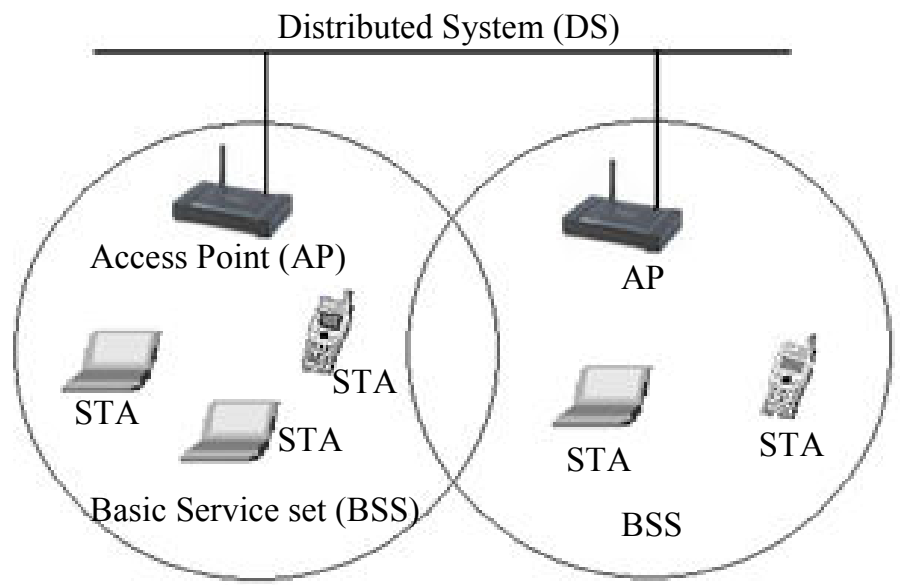

Extended Service set (ESS) - multiple cell

Fig.1 IEEE 802.11x WLAN network architecture.

\subsection{Handoff Procedures}

During mobility when a STA moves out from the coverage area of its previous AP and going to enter in a new AP's region, it breaks the link with old AP and connects to new one. This process of link transfer is simply called handoff. Handoff in link layer (L2) consists of three different phases: scanning, authentication and re-association.

a) Scanning: It is the process of searching free channels available in new APs. STA scans for APs by either sending Probe Request message (Active Scanning) or by listening to Beacon message (Passive Scanning).

b) Authentication: After scanning all channels an AP is selected by the STA using RSS indication, link quality. Transfer of credentials of STA occurs from old AP to new AP. By Authentication Request 
message the new AP either accepts or rejects the identity of the STA requesting access.

c) Re-association: It is the process for transferring association from old AP to new AP. Re-association frame includes information regarding the new association ID and supported data rule and also coordinates the forwarding of data that may still reside in the buffer of the old AP waiting for transmission to the STA.

Fig. 2 shows the complete handoff process. The overall handoff delay is the summation of scanning delay, authentication delay and re-association delay. According to [2] $90 \%$ of total handoff delay comes from scanning delay. By reducing scanning delay we can reduce handoff delay greatly. The range of scanning delay can be analyzed by (1).

$$
N \times T_{\min } \leq T_{\text {scan }} \leq N \times T_{\max }
$$

Where $N$ is the total number of channels. $T_{\min }$ is MinChannel Time and $T_{\max }$ is the MaxChannel Time. $T_{\text {scan }}$ is the total scanning delay.

\section{RELATED WORKS}

A lot of researches have been dedicated to improve the handoff performances in IEEE 802.11 based networks [2-10]. They proposed new algorithms or new protocols. For a QoS demanding applications like VoIP and multimedia seamless handover in mobility support has become a great issue. Total handoff process is divided into three steps as scanning, authentication and re-association, which are described early. As scanning process takes maximum time, most of the works are dedicated to resolve this problem. They proposed some effective scanning processes to reduce scanning delay.

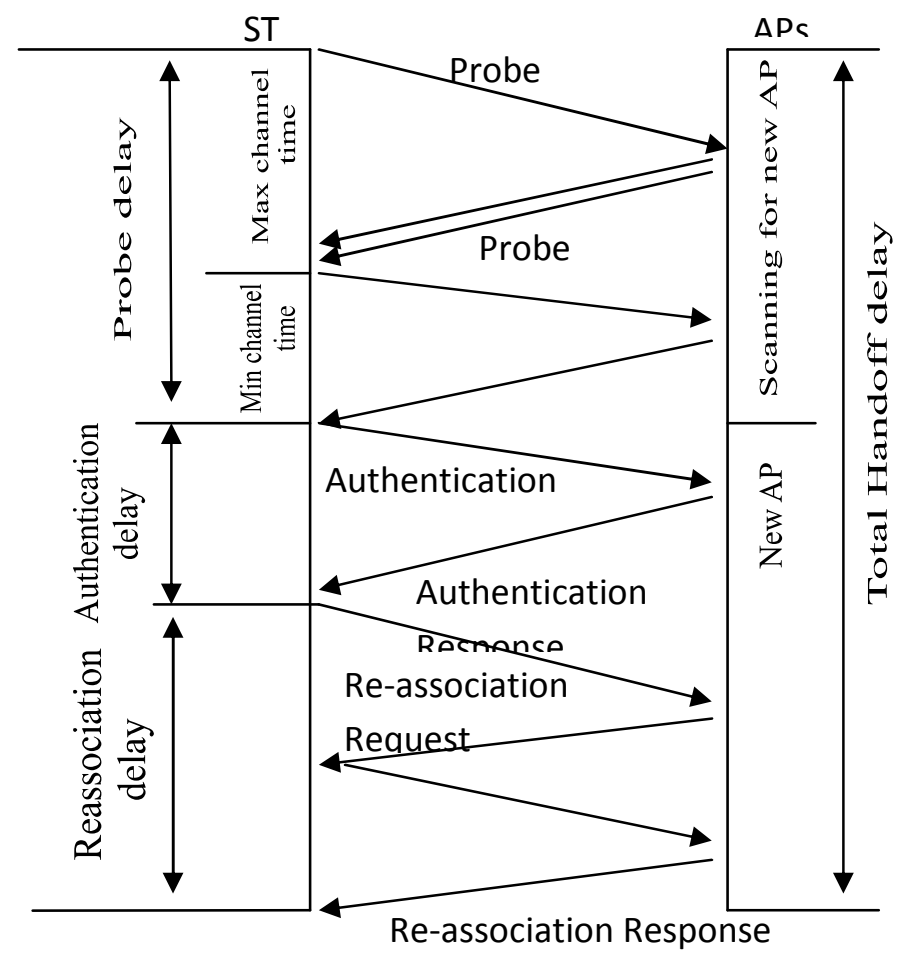

Fig. 2 Handoff procedure
In [5] H.S. Kim et al proposed selective channel scanning mechanism. They use neighbor graphs. Neighbor graph is short scan algorithm which can give the nearest APs and their channels information. They need changes in network infrastructure and use of IAPP. They considered the scanning delay as 'the time from first probe request message to last probe response message'. They neglect client's processing delay.

In [2], they take this into consideration as it has a significant role in total scanning delay. As the number of probe request messages increases, client's processing delay also increases.

Chung-Sheng Li et al. in [6] focused on neighbor graph caching mechanism for link layer handover. They use cache BSSIDs, SSIDs and channels of APs information or fast handoff mechanism Some researchers used GPS based technology to handle the mobility information and mobile node's velocity to reduce ping-pong effect and handoff delay [8].

\section{PROPOSED WORK}

Here we proposed a pre-authentication based on relative signal strength (RSS) indicator and neighbor graph information (NG) to reduce handoff delay which causes data loss and forced call termination.

For our proposed work we consider the coverage area of an AP as regular hexagonal cell. We take two access points into our account to explain our proposed approach. Fig xx describes the scenario between two APs and notations used describe the following parameters.

We divide our proposed work into three sections:

1. Handoff Detection based on RSS.

2. Neighbor Graph Algorithm.

3. Handoff Execution.

\subsection{Handoff Detection based on RSS}

Handoffs are expensive and power consuming to execute so unnecessary handoffs should be avoided. Due to false handoff initiation in the overlapping region of two APs, the connection might be handed back and forth several times between them (ping-pong effect). Moreover unreliable and inefficient handoff procedures reduce the quality and reliability of the system.

Decision of handoff can be made, based on certain level of RSS value. In this method the STA continuously monitors the signal strength and quality from the old AP and new APs. When certain range of RSS is met the STA checks the best channel and send handoff request. This is known as Mobile assisted handoff. RSS measurement based handoff detection is widely used because it is easy to implement.

We propose two levels of RSS value $S_{\mathrm{n}}$ and $S_{\text {th }}$ to determine handoff initiation time. Where $S_{\mathrm{n}}$ is the minimum required 
signal strength to maintain a connection with an AP and $S_{\text {th }}$ is the threshold value of RSS to initiate the handover process.

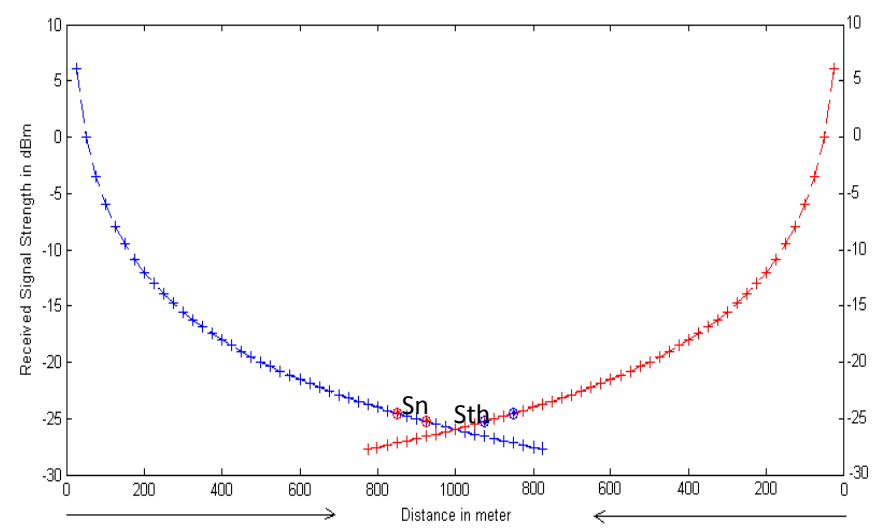

Fig. 3 Signal strength vs distance

\subsection{Neighbor Graph Algorithm}

The Neighbor Graph (NG) is an undirected graph [5], whose each edge represents a mobility path between APs. For a given edge the neighbors of it represent the set of potential next APs. Physical topology of a wireless network and the corresponding $\mathrm{NG}$ is shown in figure below [4].

The NG is defined as [2]

$\mathrm{G}=(\mathrm{V}, \mathrm{E})$

$\mathrm{V}=\left\{a p_{1}, a p_{2}, \ldots \ldots \ldots \ldots \ldots \ldots . . . a p_{i}\right\}$

$\mathrm{e}=\left(a p_{i}, a p_{j}\right)$,

$\mathrm{NC}\left(a p_{i}\right)=\left\{a p_{i k}: a p_{i k} \in \mathrm{V},\left(a p_{i}, a p_{i k}\right) \in \mathrm{E}\right\}$,

where $\mathrm{G}$ is the data structure of $\mathrm{NG}, \mathrm{V}$ is the set containing all APs, E is the set which consists of edge (e), and $\mathrm{N}$ is the neighbor APs of an AP. But our method uses not only the topological information of APs but also channels and SSID information. Thus, we modify the data structure of the NG and proposed a new algorithm:

$\mathrm{G}^{\prime}=\left(\mathrm{V}^{\prime}, \mathrm{E}\right)$

$\mathrm{V}^{\prime}=\left\{v_{i}: v_{i}=\left(a p_{i}, B S S I D, S S I D\right.\right.$, channel and $\left.\left.T_{l}\right), v_{i} \in V\right\}$, $\mathrm{e}=\left(a p_{i}, a p_{j}\right)$,

$\mathrm{NC}\left(a p_{i}\right)=\left\{a p_{i k}: a p_{i k} \in \mathrm{V}^{\prime},\left(a p_{i}, a p_{i k}\right) \in \mathrm{E}\right\}$,

Where $G^{\prime}$ is the modified NG and $V^{\prime}$ is the set containing APs with BSSIDs, SSIDs and channels and traffic load $\left(T_{l}\right)$ of neighbor APs.

$E$ is the set of edges. $N C$ is the neighbor APs of an AP. By this process they significantly reduce the handoff delay. It saves time as NGC consists of APs, channels and additional information of BSSIDs and SSIDs.

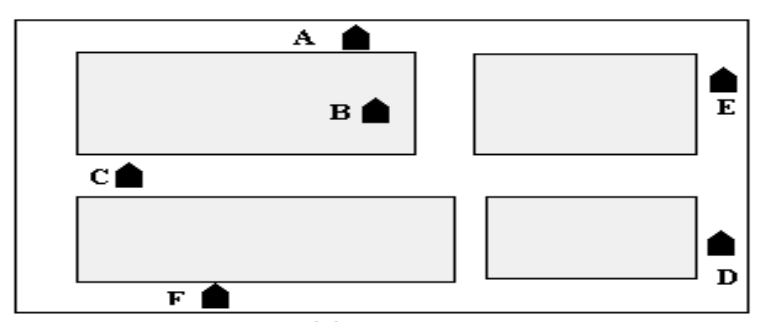

(a)

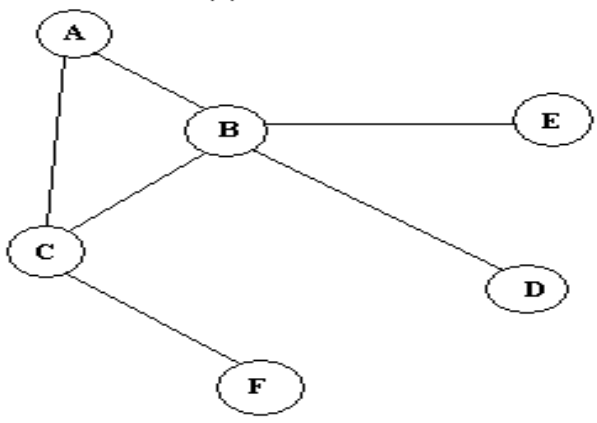

(b)

Fig. 4 Neighbor graph

\subsection{Handoff Execution}

We propose a pre-authentication method to take proper decision of handoff initiation in the overlap region of the two APs. Proper handoff initiation can reduce power consumption, ping-pong effect and packet loss during the migration of an STA from the coverage area of one AP to another AP. When the STA needs handoff it starts the NG algorithm, the NG server collects the NG information and save it to cache and gives it to the STA when it needs. The STA can know how many channels and which channels are being used by the neighboring APs and the traffic load on those channels. BSSID and SSID information are also collected to locate the neighbor APs easily. Our proposed approach is given in the following steps.

i. After call setup the STA monitors the signal quality and measures RSS continuously.

ii. When the STA enters in the overlap region it gets neighboring AP's signal.

iii. If new AP's $R S S \geq S_{\mathrm{n}}$ the $\mathrm{NG}$ algorithm starts and NG server save the information in cache. This process runs iteratively till $R S S=S_{\text {th }}$.

iv. STA fetch NG information and if new AP is present it sends beacon message to that AP.

v. The new AP's beacon kernel table updates with SSID information of STA.

vi. When old AP's $R S S=S_{\text {th }}$ the STA read the NG information again.

vii. The STA selects the best AP and send handoff initiation request. Otherwise it scans again using NG. 


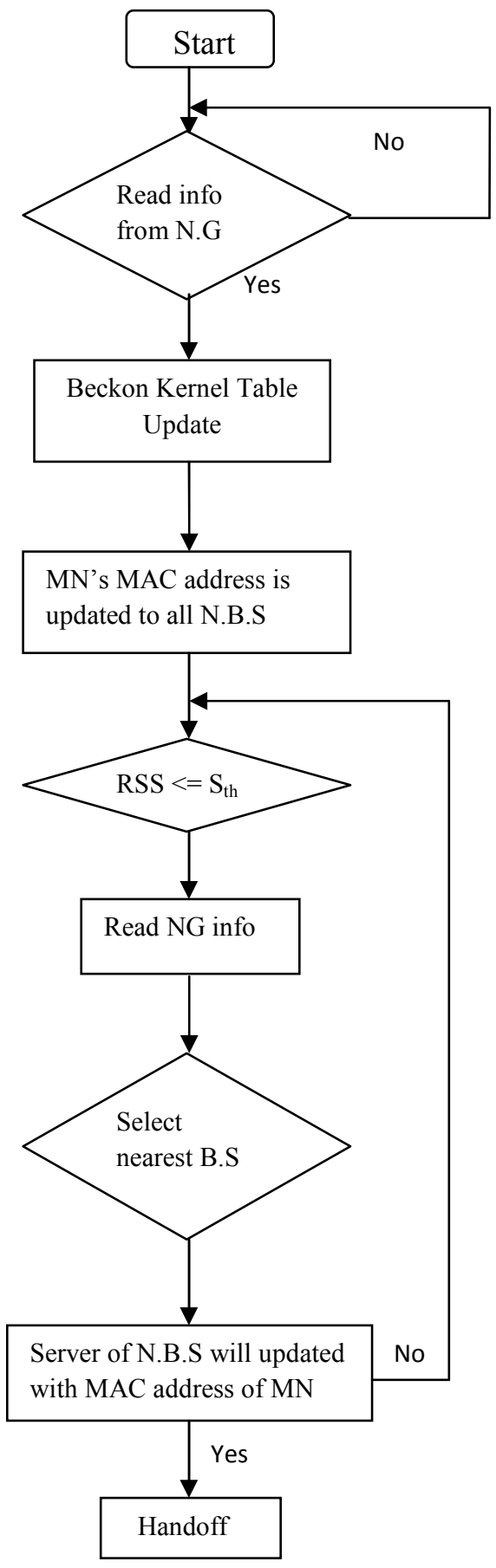

Fig. 5 Flow chart of handoff execution: NG client

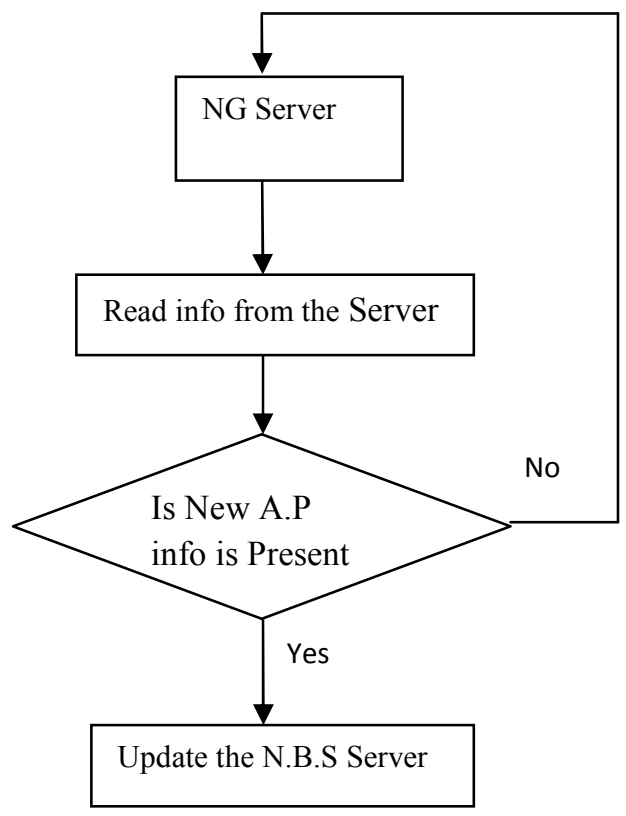

Fig. 6 Flow chart of handoff execution: NG server

\section{Result}

We assume a cellular system where each cell or coverage region of AP is regular hexagon. In an urban area the cell size may vary from few meters (micro cell) to $1 \mathrm{~km}$ (macro cell) for a standard IEEE $802.11 \mathrm{x}$ wireless networks. For our simulation we consider the following specifications of the network elements (Table 1)

The figure [7] shows simulation scenario where AP0 is call originating cell and AP1 and AP2 is two neighboring cells and random mobility path of the mobile node (STA). When the STA gradually moves towards boundary of AP0 the signal strength decreases. When it enters in the overlap region AP0-AP1-AP2, $\mathrm{NG}$ algorithm starts if RSS of neighboring cells attain $-65 \mathrm{dBm}$ $\left(\mathrm{S}_{\mathrm{n}}\right)$ and hand off occurs when the threshold of RSS reaches to $64 \mathrm{dBm}\left(\mathrm{S}_{\mathrm{th}}\right)$. These mobility and signal strength patterns are given in the tabular form (Table 2).

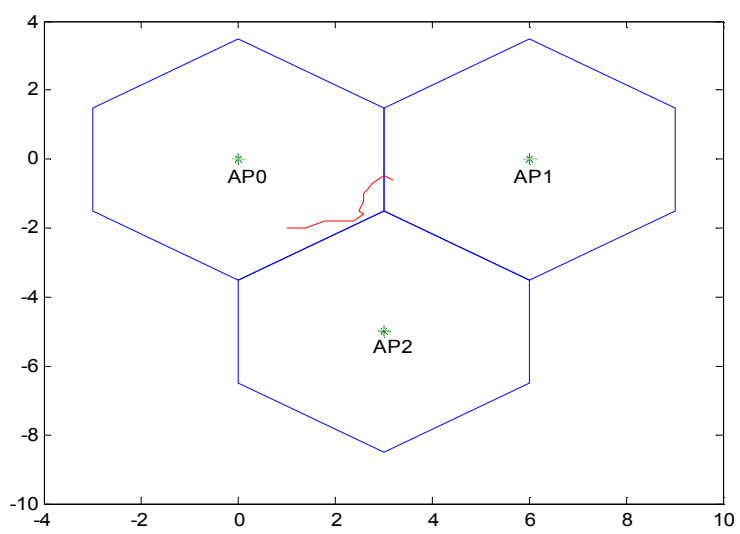

Fig. 7 Mobility of STA 
Table. 1

\begin{tabular}{|l|}
\hline Base Station antenna specification \\
\hline Frequency Range : $2.4-2.483 \mathrm{GHz}$ \\
\hline Gain: $2 \mathrm{dBi}$. \\
\hline Max Transmitter power: $15 \mathrm{dBm}$ \\
\hline Antenna height: $15 \mathrm{~m}$ \\
\hline Polarization: vertical \\
\hline $\begin{array}{l}\text { STA antenna specification (2.4GHz Omni Directional } \\
\text { wireless antenna) }\end{array}$ \\
\hline Frequency Range : $2.4-2.483 \mathrm{GHz}$ \\
\hline Gain: $1 \mathrm{dBi}$. \\
\hline Max Power: $10 \mathrm{dBm}$ \\
\hline Polarization: vertical \\
\hline
\end{tabular}

Table . 2 Simulation data for distance vs RSS

\begin{tabular}{|c|c|c|c|c|c|c|}
\hline \multirow{2}{*}{$\begin{array}{r}\text { Position } \\
(\mathbf{x} 0, \mathbf{y 0})\end{array}$} & \multicolumn{2}{|c|}{ AP0 } & \multicolumn{2}{|c|}{ AP1 } & \multicolumn{2}{|c|}{ AP2 } \\
\hline & $\begin{array}{l}\text { Distance } \\
(1 \text { unit= } \\
125 / 3 \mathrm{~m})\end{array}$ & $\begin{array}{l}\text { RSS } \\
\text { dBm) }\end{array}$ & Distance & $\begin{array}{c}\text { RSS } \\
(- \\
\text { dBm) }\end{array}$ & Distance & $\begin{array}{c}\text { RSS } \\
(- \\
\text { dBm) }\end{array}$ \\
\hline $1,-2$ & 2.2361 & 61.61 & 5.3852 & 69.24 & 3.6056 & 65.76 \\
\hline $1.2,-2$ & 2.3324 & 61.98 & 5.2 & 68.94 & 3.4986 & 65.49 \\
\hline $1.4,-2$ & 2.4413 & 62.37 & 5.016 & 68.63 & 3.4 & 65.25 \\
\hline $1.8,-1.8$ & 2.5456 & 62.74 & 4.5695 & 67.82 & 3.4176 & 65.29 \\
\hline $2,-1.8$ & 2.69067 & 63.22 & 4.3863 & 67.46 & 3.3526 & 65.13 \\
\hline $2.2,-1.8$ & 2.8425 & 63.69 & 4.2048 & 67.09 & 3.2985 & 64.98 \\
\hline $2.4,-1.8$ & 3 & 64.16 & 4.0249 & 66.72 & 3.2558 & 64.87 \\
\hline $2.6,-1.6$ & 3.0529 & 64.32 & 3.7577 & 66.12 & 3.4234 & 65.31 \\
\hline $2.5,-1.5$ & 2.9155 & 63.92 & 3.8079 & 66.23 & 3.5355 & 65.59 \\
\hline $2.6,-1.2$ & 2.8636 & 63.76 & 3.6056 & 65.76 & 3.8210 & 66.26 \\
\hline $2.6,-1$ & 2.7857 & 63.52 & 3.544 & 65.61 & 4.02 & 66.7 \\
\hline $2.8,-0.7$ & 2.8862 & 63.83 & 3.2757 & 64.93 & 4.3046 & 67.29 \\
\hline $3,-0.5$ & 3.0414 & 64.28 & 3.0414 & 64.28 & 4.5 & 67.68 \\
\hline $3.2,-0.6$ & 3.2558 & 64.87 & 2.8636 & 63.76 & 4.4 & 67.49 \\
\hline
\end{tabular}

The table observation shows that when the $\mathrm{MN}$ is at $(2.2,-1.8)$ the RSS of AP2 reaches $-64.9872 \mathrm{dBm}$ and NG algorithm for overlap region Ap0-AP2 starts but the RSS decreases gradually afterwards so the hand off does not take place between AP0 and AP2. When the MN is at

$(2.8,-0.7)$ the RSS of AP1 reaches $-64.9270 \mathrm{dBm}$ and NG algorithm for overlap region Ap0-AP1 starts and as the RSS gradually reaches to $-64 \mathrm{dBm}$ the hand off occurs between AP0 and $\mathrm{AP} 1$ and $\mathrm{MN}$ is connected to $\mathrm{AP} 1$ after discarding the connection with AP0.

\section{CONCLUSIONS}

In this article we have discussed a handoff method using neighbor graph cache algorithm to reduce the scanning delay. We consider the RSS values and NG information to take right decision of handoff initiation process during mobility of STA. By taking the right decision of handoff initiation time we can reduce the handoff delay and handoff failure probability. Our simulation results show that the proposed method is efficient and support seamless handoff management in wireless local area networks.

\section{REFERENCES}

[1] Wankawee Puangkor and Panita Pongpaibool. 'A Survey of Techniques for Reducing Handover Latency and Packet Loss in Mobile IPv6'. IM 20060306.

[2] A Mishra, M Shin and W Arbaugh, "An empirical analysis of the IEEE 802.11 MAC layer handoff process", ACM SIGCOMM Comp Comm Rev. vol.33, no.2,April . 2003.

[3] Ping-Jung Huang and Yu-Chee Tseng. 'A Fast Handoff Mechanism for IEEE 802.11 and IAPP Networks'.Vehicular Technology conference, 2006.VTC 2006-Spring.IEEE 63 ${ }^{\text {rd }}$, vol. 2, pp 966-970.

[4] Eunsoo Shim, Hung-yu Wei, Yusun Chang, and Richard D. Gitlin. "Low Latency Handoff for Wireless IP QOS with Neighbor Caching”. Columbia University Technical report.

[5] H-S Kim et. al. "Selective channel scanning for fast handoff in Wireless LAN using neighbor graph" International Technical Conference on Circuits/Systems, Computers and Communication. Japan July 6-8, 2004.

[6] C-S. Li et.al. 'A Neighbor Caching mechanism for Handoff in IEEE 802.11 Wireless Networks.' Springer 20 March 2008, DOI 10.1007/s11227-008-0175-3.

[7] Sangho Shin et.al. 'Reducing MAC Layer Handoff Latency in IEEE 802.11 Wireless LANs'.MobiWac'04, Oct. 2004. Pennsylvania USA.

[8] Chien-Chao Tseng, K-H Chi, M-D Hsieh and H-H Chang," Location-based Fast Handoff for 802.11 Networks",IEEECommunication Letters,vol.9, no.4 April 2005.

[9] A. Mishra, M. H. Shin, and W. Albaugh, "Context Caching using Neighbor Graphs for Fast Handoff in a Wireless ," Computer Science Technical Report CSTR-4477, 2003.

[10] IEEE "Recommended Practice for Multi-Vendor Access Point Interoperability via an Inter-Access Point Protocol Across Distribution Systems Supporting IEEE 802.1 Operation," IEEE Standard 802.11, 2003. 